\title{
Postoperatively altered total clearance of ascorbic acid in surgical tumour patients
}

\author{
Andreas Rümelin, Ulrich Fauth, Heiko Hübner and Miklós Halmágyi
}

Klinik für Anästhesiologie, Johannes Gutenberg Universität Mainz, Germany

\begin{abstract}
A bstract
Background: Postoperatively lowered ascorbic acid (AA) plasma concentration is postulated to be due to an increased perioperative activity (radical scavenging activity, cofactor for enzymes, etc) and consumption in response to surgical trauma.

Objective: To test this hypothesis by measuring total clearance $\left(\mathrm{Cl}_{\mathrm{tot}}\right)$ of AA in plasma. Any alteration in AA consumption would be due to an altered $\mathrm{Cl}_{\text {tot }}$ of $\mathrm{AA}$ in plasma. Unaltered postoperative $\mathrm{Cl}_{\text {tot }}$ would exclude the postulated theory of an increased perioperative AA consumption.

Design: The $\mathrm{Cl}_{\text {tot }}$ of $\mathrm{AA}$ in plasma was calculated for 17 tumour patients undergoing major maxillofacial surgery on the first and third postoperative days compared with the preoperative values after bolus injection of $4 \mathrm{mg} \mathrm{kg}^{-1}$ body weight AA was given intravenously. Blood samples were taken before and 15, 30, 45, 60 and $120 \mathrm{~min}$ after injection. AA in plasma was analysed by high-performance liquid chromatography. Results: The concentration of $\mathrm{Cl}_{\text {tot }}$ [median $\left(25 \% / 75 \%\right.$ percentile)] was $10.2(7.9 / 12.5) 1 \mathrm{~h}^{-1}$ preoperatively. On the first and third postoperative days [median: $17.6(10.9 / 27.9)$ and $15.3(12.1 / 30.5) 1 \mathrm{~h}^{-1}$, respectively] the $\mathrm{Cl}_{\text {tot }}$ was significantly increased $(p<0.05)$.

Conclusions: $\mathrm{Cl}_{\text {tot }}$ was significantly increased in postoperative patients compared with preoperative values. These results are consistent with the postulated increase in perioperative AA consumption.
\end{abstract}

Keywords: ascorbic acid, operation, surgery, vitamin $C$.

Received: 3I May 2002; Revised: 19 July 2002; Accepted: 23 August 2002

\section{Introduction}

A postoperatively lowered plasma concentration of ascorbic acid (AA) has been shown previously (15) and is interpreted as part of oxidative stress. Oxidative stress is defined as an imbalance of increased pro-oxidants (radicals, etc.) and reduced antioxidants (vitamins, etc.) (6). It is postulated that the reduced antioxidants are the result of increased perioperative AA activity (radical scavenging activity, cofactor for enzymes, etc.). Increased perioperative AA activity would be accompanied by altered pharmacokinetics of AA. The term AA activity is used in this context to describe the clearance of AA from blood (total clearance $\left.=\mathrm{Cl}_{\mathrm{tot}}\right) \cdot \mathrm{Cl}_{\mathrm{tot}}$ is influenced by the renal $\left(\mathrm{Cl}_{\text {renal }}\right)$ and metabolic $\left(\mathrm{Cl}_{\text {meta }}\right)$ AA consumption. Therefore, AA activity is not identical to AA consumption.

At present, no data on AA clearance in postoperative patients are available. To investigate this, the preoperative and postoperative $\mathrm{Cl}_{\text {tot }}$ of $\mathrm{AA}$ were calculated. Values of preoperative and postoperative $\mathrm{Cl}_{\text {tot }}$ that were not significantly different would exclude the postulated increased perioperative AA activity.

\section{Materials and methods}

\section{Patients}

The investigation was carried out according to the recommendations for clinical trials in humans in the Declaration of Helsinki and was approved by the Ethics Committee of the University of Mainz.

Seventeen patients $(62 \pm 13.3$ years old, body mass index $22.8 \pm 3.2 \mathrm{~kg} \mathrm{~m}^{-2}$ ) were hospitalized for major maxillofacial tumour operations (12 for epithelial carcinoma, 3 for squamous cell carcinoma and 2 for basal cell carcinoma). Ten patients had preoperative radiotherapy and/or chemotherapy until some weeks before hospitaliza- 
tion, whereas seven patients had no neoadjuvant therapy. The average duration of operation was $525 \pm 138$ min (mean \pm SD). All patients were admitted to the intensive care unit (ICU) postoperatively. None of the patients showed any postoperative complications within the study period. Patients with at least one of the following factors were excluded from the study: pregnant patients, not fully mentally capable patients, and patients with a known history of allergic reactions to one of the substances in the AA ampoules, urethrolithiasis, ureterolithiasis, haemochromatosis, thalassaemia or any other tendency to haemolytic reactions.

\section{Materials}

The AA ampoules used were Vitamin C Injektopas

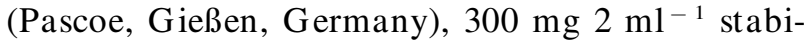
lized in sodium hydrogen carbonate.

\section{Experimental procedure}

All patients were studied on the day before operation (P) and on the first (D1) and third (D3) postoperative days. During each investigation blood samples were taken as baseline values of AA. Subsequently, a bolus of $4 \mathrm{mg} \mathrm{kg}^{-1}$ body weight $\mathrm{AA}$ was given intravenously. As Levine et al. (7) described in their depletion-repletion study, these amounts are sufficient to influence pharmacokinetics without major adverse effects. Blood samples were taken $15,30,45$, 60 and $120 \mathrm{~min}$ after bolus injection. A previously published method was used to analyse AA in plasma (8). In brief, all samples were collected in tubes with $1.6 \mathrm{~g}^{-1}$ ethylenediaminetetra-acetic acid (EDTA) as anticoagulant. During the transport of the specimen to the laboratory they were stored on ice in darkness. Plasma was obtained by centrifugation, and cold $10 \%$ perchloric acid containing $1 \%$ metaphosphoric acid in brown microcentrifuge tubes was added for deproteinization. All samples were kept at $4^{\circ} \mathrm{C}$ for $60 \mathrm{~min}$ to complete the deproteinization process, followed by centrifugation. Subsequently, a mobile phase was added (1:1), and the samples were centrifuged, filtered and stored in liquid nitrogen until the analysis was carried out. All samples were analysed in duplicate by high-performance liquid chromatography with ultraviolet detection.

The $\mathrm{Cl}_{\text {tot }}$ of $\mathrm{AA}$ after bolus injection was then investigated. $\mathrm{Cl}_{\text {tot }}$ is defined as dose/area under the curve (AUC). The AUC was obtained directly from the data (semi-log trapezoidal rule) above baseline and after extrapolation of the data to the moment of bolus injection. The residual AUC was calculated according to $\mathrm{P} 6 / k$, where $\mathrm{P} 6$ is the last measured plasma concentration and $k$ is the elimination rate constant.

\section{Statistical analysis}

Parameters were evaluated for normality using the Shakiro-Wilk test. Differences between postoperative parameters and preoperative baseline values were evaluated using the two-sided Student's $t$-test for parametric data and the Wilcoxon rank-sum test for skewed data. Sigma Stat ${ }^{\circledR}$ was used during this statistical analysis. For sample size determination the standard deviation of the AUC differences $(\Delta$ AUC of P and D1) were calculated. The preoperative AUC was calculated at $28 \mathrm{mg}^{-1}$ per hour. To increase the probability that the relative difference was clinically relevant, the relative difference was set to $50 \%$ of preoperative AUC (14 $\mathrm{mg}^{-1}$ per hour). The level of probability was set to $5 \%$ and $\beta$-error to $10 \%$. Under these conditions a minimum of 12 patients needed to be included in the study to achieve the necessary statistical power.

\section{Results}

\section{Ascorbic acid in plasma}

The postoperative AA concentration in plasma was below the reference range $\left(30.2-102.5 \mu \mathrm{mol} \mathrm{l}^{-1}\right)$ (9) on D 1 and D3 [median (25\%/75\% percentile): 24.4 $(14.3 / 35)$ and $33.4(20.2 / 44.5) \mu \mathrm{mol} 1^{-1}$, respectively). Compared with the median of $25.6 \mu \mathrm{mol} 1^{-1}$ for the preoperative values $(25 \% / 75 \%$ percentile: $\left.15.1 / 54.5 \mu \mathrm{mol} \mathrm{l}^{-1}\right)$, no significant differences were seen postoperatively.

\section{A rea under the curve and total clearance of ascorbic acid}

The preoperative AUC (P) $\left(29.6 \pm 15.3 \mathrm{mg} \mathrm{l}^{-1}\right.$ per hour) and postoperative AUC (D 1$)(14.2 \pm 14.7 \mathrm{mg}$ $1^{-1}$ per hour) was calculated for the determination of the sample size and $\mathrm{Cl}_{\text {tot }}$.

The median $\mathrm{Cl}_{\text {tot }}$ was $10.2(7.9 / 12.5) 1 \mathrm{~h}^{-1}$ preoperatively. On D1 and D 3 the $\mathrm{Cl}_{\text {tot }}$ was significantly increased $(p<0.05)$, to $17.6(10.9 / 27.9)$ and 15.3 $(12.1 / 30.5) 1 \mathrm{~h}^{-1}$, respectively (Fig. 1, Table 1).

\section{Discussion}

Postoperatively low concentrations $(<30.2 \mu \mathrm{mol}$ $1^{-1}$ ) of AA in plasma have been described previously (9). Low AA concentrations are common in ICU patients $(4,10)$ and the influence of low AA concentrations on the onset of postoperative com- 


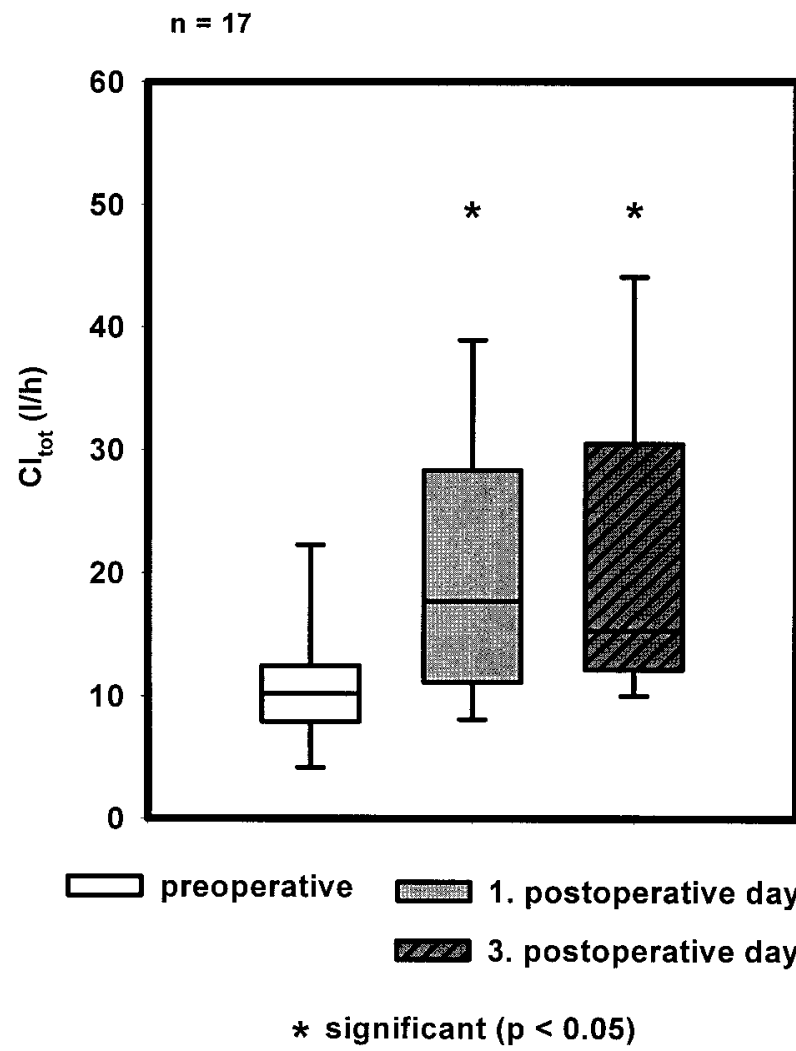

Fig. 1. Total clearance $\left(\mathrm{Cl}_{\mathrm{tot}}\right)$ of ascorbic acid in plasma preoperatively and on the first and third postoperative days. Data are presented as medians with $25 \%$ and $75 \%$ percentiles.

plications in ICU patients has been discussed (11, 12). Although it seems that the low AA plasma concentration is clinically relevant, the underlying mechanism is not totally clear.

Previously, reduced postoperative AA concentration in plasma was discussed as being a result of perioperative haemodilution. Several studies attempted to exclude haemodilution as a factor by the documentation of the haematocrit in plasma (13) or AA concentration in the buffy layer (1). The haematocrit or haemoglobin level is a useful marker to exclude haemodilution in patients without parenteral administration of blood products (e.g. myocardial infarction), but in perioperative situations, where patients may be given blood products, the haemoglobin is only a marker for an adequate substitution (corrected haemoglobin concentration) and not for haemodilution.

Despite the absence of any pharmacokinetic studies that definitely reject the haemodilution theory and show a perioperative increase in the metabolic activity of AA, in the recent years the low postoperative AA plasma concentration has been postulated to be the result of increased consumption of AA due to non-nutritive effects (cofactor for enzymes, radical scavenging activity, etc.) in response to surgical trauma. To test this hypothesis the $\mathrm{Cl}_{\text {tot }}$ was calculated preoperatively and postoperatively. Any alteration in metabolic or renal AA consumption would lead to an alteration in $\mathrm{Cl}_{\text {tot }}$. Preoperative and postoperative $\mathrm{Cl}_{\text {tot }}$ values that were not significantly different would exclude the postulated theory of increased perioperative AA consumption.

Table I. Area under the curve (AUC) and total clearance $\left(\mathrm{Cl}_{\text {tot }}\right)$ of ascorbic acid in plasma preoperatively and on the first (DI) and third (D3) postoperative days

\begin{tabular}{|c|c|c|c|c|c|c|}
\hline & \multicolumn{2}{|l|}{ Preoperative } & \multicolumn{2}{|l|}{ DI } & \multicolumn{2}{|l|}{ D3 } \\
\hline & AUC (mg I- ${ }^{-1}$ per hour) & $\mathrm{Cl}_{\text {tot }}\left(\mathrm{Ih}^{-1}\right)$ & AUC (mg I- ${ }^{-1}$ per hour) & $\mathrm{Cl}_{\text {tot }}\left(\mathrm{Ih} \mathrm{h}^{-1}\right)$ & AUC (mg I- ${ }^{-1}$ per hour) & $\mathrm{Cl}_{\text {tot }}\left(\mathrm{I} \mathrm{h} \mathrm{h}^{-1}\right)$ \\
\hline PI & 30.2 & 10.2 & 4.5 & 69.0 & 6.9 & 44.8 \\
\hline P2 & 68.5 & 3.9 & 23.9 & II.I & 36.3 & 7.3 \\
\hline P3 & 25.1 & 8 & 19.8 & 10.1 & 20.1 & 9.9 \\
\hline P4 & 34.9 & 7.8 & 24.4 & 11.2 & 15.7 & 17.3 \\
\hline P5 & 46.8 & 5.3 & 6.4 & 39.1 & 7.9 & 31.7 \\
\hline P6 & 20 & 12.7 & 32.2 & 7.9 & 18.6 & 13.7 \\
\hline P7 & 32.3 & II.I & 12.3 & 29.3 & 8.7 & 41.2 \\
\hline P8 & 29.5 & 8.1 & 13.6 & 17.6 & 13.7 & 17.5 \\
\hline P9 & 60.2 & 3.7 & 29.7 & 7.4 & 20.8 & 10.6 \\
\hline PIO & 28.1 & 9.3 & 17.7 & 14.7 & 8.6 & 30.1 \\
\hline PII & 24.9 & 9.6 & 13.5 & 17.8 & 5.1 & 47.4 \\
\hline $\mathrm{PI} 2$ & 22.4 & 13.4 & 7.0 & 37.3 & 26.4 & 11.4 \\
\hline $\mathrm{PI} 3$ & 9.5 & 26.9 & 12.1 & 21.0 & 17.7 & 14.4 \\
\hline PI4 & 22.7 & 11.9 & 22.4 & 12.0 & 21.8 & 12.4 \\
\hline PI5 & 19.4 & 12.4 & 13.5 & 17.8 & 16.9 & 14.2 \\
\hline PI6 & 16.5 & 12.1 & 11.5 & 17.5 & 11.6 & 17.3 \\
\hline PI7 & 12.2 & 24.5 & 11.0 & 27.4 & 19.7 & 15.3 \\
\hline
\end{tabular}


In this study a preoperative $\mathrm{Cl}_{\text {tot }}$ of $10.2 \mathrm{~h} \mathrm{~h}^{-1}$ was calculated. Similar clearance was reported by Levine et al. (7) in healthy volunteers. Postoperatively, a significant increase in $\mathrm{Cl}_{\text {tot }}$ was seen on the first and third postoperative days. In addition, the postoperatively altered AA clearance seemed to be independent of preoperative AA plasma values. Even low preoperative AA plasma concentrations led to increased postoperative $\mathrm{Cl}_{\text {tot }}$ in this study.

In general, $\mathrm{Cl}_{\text {tot }}$ is influenced by $\mathrm{AA}$ metabolism $\left(\mathrm{Cl}_{\text {meta }}\right)$ and elimination $\left(\mathrm{Cl}_{\text {renal }}\right)$. In theory, the increased $\mathrm{Cl}_{\text {tot }}$ seen here could be an effect of increased AA clearance by the kidney $\left(\mathrm{Cl}_{\text {renal }}\right)$.

The $\mathrm{Cl}_{\text {renal }}$ is known to be dependent on the plasma concentration of AA. AA is actively excreted in urine until a threshold value is reached at the plasma concentration (14). In this study, preoperative and postoperative AA values in plasma were below the reference range and not significantly different. Therefore, an unchanged renal clearance was postulated in these postoperatively uncomplicated patients on the first and third postoperative days.

These data show an increased postoperative total clearance of AA in the blood compared with preoperative values. It could not be shown from the data whether the rise in $\mathrm{Cl}_{\text {tot }}$ was an effect of increased renal function $\left(\mathrm{Cl}_{\text {renal }}\right)$ or metabolic function (non-nutritive effects of $\mathrm{AA})\left(\mathrm{Cl}_{\text {meta }}\right)$. Further studies are necessary to differentiate between these functions.

\section{Acknowledgements}

The authors thank Prof. Dr E. Jähnchen, Department of Clinical Pharmacology, Bad Krozingen, Germany, and $\mathrm{Mr}$ Rippin, Institute for Medical Statistics, University of Mainz, Germany. The results of this study are part of $\mathrm{H}$. Hübner's dissertation.

\section{References}

1. Vallance $S$. Changes in plasma and buffy layer vitamin C following surgery. Br J Surg 1988;75:366-70.

2. Kreinhoff U, Elmadfa I, Salomon F, Weidler B. Untersuchungen zum Antioxidantienstatus nach operativem Stress. Infusionstherapie 1990;17:261-7.

3. Dingchao H, Zhiduan Q, Liye H, Xiaodong F. The protective effects of high-dose ascorbic acid on myocardium against reperfusion injury during and after cardiopulmonary bypass. Thorac Cardiovasc Surg 1994;42:276-8.

4. Schorah CJ, Downing C, Piripitsi A et al. Total vitamin $\mathrm{C}$, ascorbic acid, and dehydroascorbic acid concentrations in plasma of critically ill patients. Am J Clin Nutr 1996;63:760-5.

5. Erhola M, Nieminen NM, Kellokumpu-Lehtinen $\mathrm{P}$ et al. Effects of surgical removal of lung cancer on total plasma antioxidant capacity in lung cancer patients. J Exp Clin Cancer Res 1998;17:219-25.

6. Biesalski HK, Böhles H, Esterbauer $\mathrm{H}$ et al. Antioxidant vitamins in prevention. Consensus statement. Clin Nutr 1997;16:151-5.

7. Levine M, Conry-Cantilena C, Wang Y et al. Vitamin $\mathrm{C}$ pharmacokinetics in healthy volunteers: evidence for a recommended dietary allowance. Proc Natl Acad Sci USA 1996;93:3704-9.

8. Rümelin A, Fauth U, Halmágyi M. Determination of ascorbic acid in plasma and urine by high performance liquid chromatography with ultraviolet detection. Clin Chem Lab Med 1999;37:533-6.

9. Friedrich W. Vitamin C. In: Handbuch der Vitamine. Munich, Vienna, Baltimore: Urban \& Schwarzenberg, 1987; p. 611.

10. Rümelin A, Fauth U, Halmágyi M. Reduzierte Ascorbinsäurekonzentrationen im Plasma bei intensivbehandlungspflichtigen Patienten in der frühen postoperativen Phase. Akt Ernahr Med 1999;24:267-9.

11. Goode HF, Cowley HC, Walker BE et al. Decreased antioxidant status and increased lipid peroxidation in patients with septic shock and secondary organ dysfunction. Crit Care Med 1995;23:646-51.

12. Borelli E, Roux-Lombard P, Grau GE et al. Plasma concentrations of cytokines, their soluble receptors, and antioxidant vitamins can predict the development of multiple organ failure in patients at risk. Crit Care Med 1996;24:392-7.

13. Ballmer PE, Reinhart WH, Jordan P et al. Depletion of plasma vitamin $\mathrm{C}$ but not of vitamin $\mathrm{E}$ in response to cardiac operations. J Thorac Cardiovasc Surg 1994;108:311-20.

14. Kallner AB, Hartmann D, Hornig DH. On the requirements of ascorbic acid in man: steady-state turnover and body pool in smokers. Am $J$ Clin Nutr 1981;34:1347-55.

\footnotetext{
A. Rümelin

Kinik für Anästhesiologie, Johannes Gutenberg Universität

Mainz, Langenbeckstrasse I, D-55I0I Mainz, Germany

Tel.: +49 6131 172793

Fax: +49 6131 175538

E-mail: ruemelin@mail.uni-mainz.de
} 